\title{
VALIDATION OF A BIODYNAMIC MODEL OF PUSHING AND PULLING
}

\author{
ROBERT O. ANDREs* and DON B. ChafFin $\dagger$ \\ *Department of Exercise Science, 21 Totman Building, University of Massachusetts, Amherst, \\ MA 01003, U.S.A. and +Center for Ergonomies, The University of Michigan, Ann Arbor, Michigan, \\ U.S.A.
}

\begin{abstract}
Pushing and pulling during manual material handling can increase the compressive forces on the lumbar disc region while creating high shear forces at the shoe-floor interface. A sagittal plane dynamic model derived from previous biomechanical models was developed to predict LS/S1 compreasive force and required coefficients of friction during dynamic cart pushing and pulling. Before these predictions could be interpreted, however, it was necessary to validate model predictions against independently measured values of comparable quantities. This experiment used subjects of disparate stature and body mass, while task factors such as cart resistance and walking speed were varied. Predicted ground reaction forces were compared with those measured by a force platform, with correlations up to 0.67 . Predicted erector spinae and rectus abdominus muscle forces were compared with muscle forces derived from RMS-EMGs of the respective muscle groups, using a static force build-up regression relationship to transform the dynamic RMS-EMGs to trunk muscle forces. Although correlations were low, this was attributed in part to the use of surface EMG on subjects of widely varied body mass. The biodynamic model holds promise as a tool for analysis of actual industrial pushing and pulling tasks, when carefully applied.
\end{abstract}

\section{INTRODUCTION}

Carts of various sizes, weights and configurations are frequently pushed or pulled manually in many industrial situations. Among these are the tyre manufacturing industry, the fiberglass manufacturing industry, commercial laundries, and the airline industries. A large proportion of these tasks involve the worker pulling on doors and hoses, pushing on carts, and generally attempting a task which imparts a high shearing force to the feet, with resulting slip and fall injuries (Safety Sciences, 1977). It is estimated that over $20 \%$ of the worker's compensation costs each year are a result of fall or slip related injuries in the U.S. (Szymusiac and Ryan, 1982). A study concerning a large manufacturing operation in England reported that $36-45 \%$ of back pain was caused by a slip or fall (Manning, 1983). These statistics paint a dangerous picture for workers involved with pushing or pulling tasks, which increase the risks of slipping and falling or overexerting the back.

Several investigators have addressed the performance aspects of push/pull tasks. Dempster (1958) studied static pull forces during standing. Kroemer measured maximal isometric pushing forces in 65 different positions and assessed the effects of varied foot friction during pushing (Kroemer, 1969, 1971). Ayoub and McDaniel (1974) studied the loading of the lumbar spine during static pushes and pulls against a wall, in various body postures. One Swedish study (Winkel, 1983) concerned the manual handling of food and beverage carts on wide-body airplanes and measured only the hand forces exerted on stationary carts. Several recommendations were made about cart con-

Received in final form 25 March 1991. figuration and loading as a result of this study. However, none of these studies considered the dynamic case where the worker moves during the push or pull task.

Pushing and pulling hand forces have been measured while the subject walked on a treadmill (Snook et al., 1970) with different handle heights and adjustable treadmill resistance. Strindberg and Peterson (1972) used psychophysical methods to study force perception while pushing trolleys. These studies began to approach more realistic dynamic simulations of actual industrial situations. A German group studied the load on the spine during the transport of dustbins (Jager et al., 1984). These authors utilized a simple static model of L5/S1 torques, and they also measured the EMG activity of back, leg, and hand muscles. However, no validation of their model was offered, and the EMG information for the back muscles was not compared with the L5/S1 torque predictions.

The doctoral research of Lee (Lee, 1982; Lee et al., 1989) formulated a dynamic biomechanical model of cart pushing and pulling. The inputs to the model included subject anthropometry, body postures during dynamic tasks, and hand forces exerted on the cart handle. The specific model predictions were horizontal and vertical foot forces and gross torso muscle and vertebral column loadings when pushing or pulling. Laboratory validation of the model took place with a cart simulator. Dynamic foot forces, hand forces required to move the cart, body motions at various speeds, and back muscle actions were measured while six subjects pushed or pulled the cart simulator. Foot force predictions were compared to measured (by force platform) foot forces, while predicted torso muscle forces were converted to 'equivalent' integrated electromyograms (IEMGs) and then compared 
with the measured IEMGs. These validation experiments were performed at thrce handle heights $(66,109$, $152 \mathrm{~cm}$ ) and three cart resistances (light, medium, heavy). Two speeds (approximately 1.8 and $3.6 \mathrm{~km} \mathrm{~h}^{-1}$ ) were used at only the middle handle height. The four male and two female subjects ranged in stature from 161 to $175 \mathrm{~cm}$, and the body mass range was $50-80 \mathrm{~kg}$.

Lee et al. (1989) reported the results of static calibrations performed for the dynamic experiment, but the dynamic results have not been published. The purpose of our investigation was to validate a model (based on Lee's model) in the laboratory with a broader anthropometric population.

\section{METHODS}

\section{Model development}

The biomechanical model used for this study was based on that used by Lee (1982). The mathematical relationships and assumptions which allow the prediction of the desired outputs (foot reactive forces and L5/S1 compressive forces) will be described. There were several intermediate steps before the predictions were made; these will also be examined.

Two different sets of link lengths were calculated. The distance between the joint markers (LEDs), in the sagittal plane, was measured by the position detection system. The $Y$ and $Z$ coordinates were calibrated first, corrected for distortion, and smoothed with a digital filter with optimal cut-off frequencies [determined for each data record as described by Jackson (1979)] before calculating relative link lengths (link lengths in the sagittal plane, possibly foreshortened if the link was out of plane). Absolute link lengths were based on ratios to total stature (Drillis and Contini, 1966).

The position of LS/S1 was calculated because there was no marker on that location. These calculations were based on Chaffin and Andersson (1984, Chap. 6). The link center of gravity (CG) positions were calculated with respect to relative link length. Ratios based on Dempster (1955) were used.

Link masses were calculated as a ratio to total body mass. A major assumption made at this point was that the left arm position was the same as the right arm, as viewed from the sagittal plane. When markers were placed on the inside of the left arm, they were never detected by the position detection system because they were blocked by the right arm. Therefore, the masses of the forearm and hand link and the upper limb link were doubled to represent both arms acting in the same sagittal plane position. The ratios were again from Dempster (1955).

Joint angles were calculated from joint marker coordinates with respect to the horizontal. The joint angles correspond to the angle from the horizontal of respective links (i.e. joint angle No. 1 was the angle from the horizontal of link No. 1, the forearm and hand; see Fig. 1).

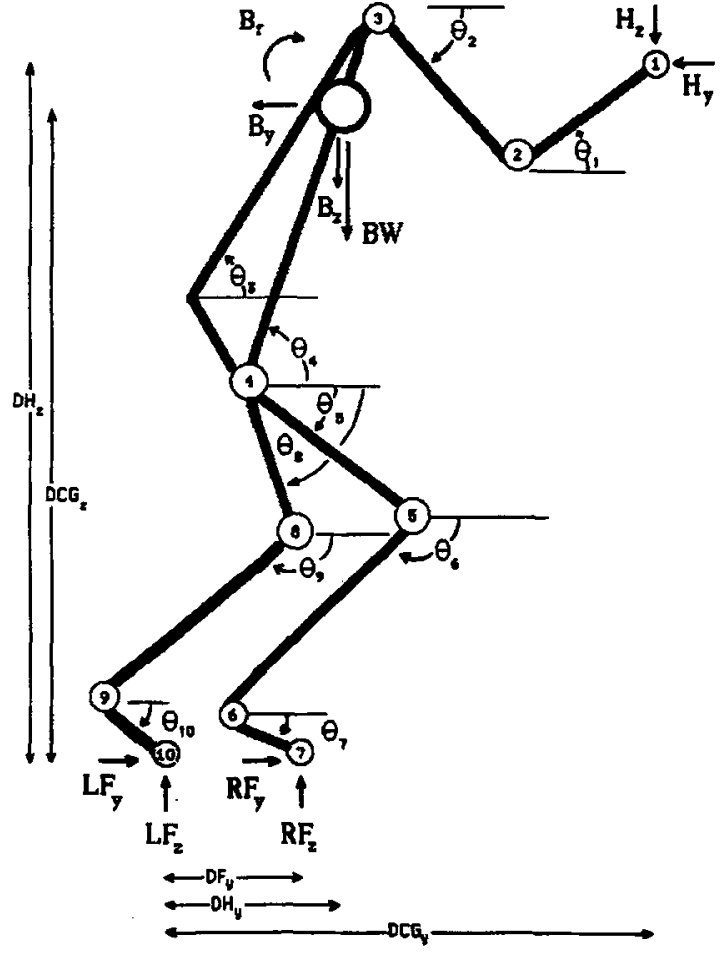

Fig. 1. Free body diagram of double support stance with joint angles used by the model and locations of joint center markers. Variables and resulting equilibrium equations are given in the text.

The whole body CG position was calculated using the segmental moments method (Miller and Nelson, 1976). The location of the upper body CG was calculated with the same approach using only links above LS/S1 (hands, forearms, upper arms, and trunk from shoulder to L5/S1).

Mass moments of inertia were calculated for each link as the product of the link mass and $\boldsymbol{K}^{2}$, where $\boldsymbol{K}$ was the product of relative link length and a constant representing the radius of gyration (Chandler et al., 1975; Plagenhoef, 1966).

Link CG, whole body CG, upper body CG, and joint angle data were differentiated twice so that the respective accelerations could be used for calculation of inertial forces. The derivatives were calculated with a finite-impulse-response digital recursive filter (Oppenheim and Schafer, 1975; Lanshammar, 1982). Optimal filter coefficients were derived for these data and subsequently applied uniformly.

The linear and rotational inertial forces resist body linear and angular accelerations. These inertial forces were calculated for each link as the negative of the product of the link mass and the linear acceleration in $Y$ and $Z$ directions. Whole body inertial forces acting at the whole body $C G$ were calculated as the negative of the product of the whole body mass and the $Y$ and $Z$ accelerations of the whole body CG. Since there was no overall body angular acceleration, the whole body rotational inertial force was calculated as the sum of the individual link rotational inertial forces. 


\section{Calculation of ground reaction forces at the feet}

Two different situations exist during normal walking gait: a single support phase, when only one foot contacts the support surface while the other foot swings through to its next placement; and a double support phase, when both feet are in contact with the support surface (see Fig. 1). Foot force calculations are detailed below for each case.

Single support. Assume:

(1) two arms act as one (sum $Y$ and $Z$ forces from the separate handles);

(2) quasi-static equilibrium,

$$
\begin{aligned}
& \Sigma F_{y}=0=R F_{y}-H_{y}-B_{y}, \\
& R F_{y}=H_{y}+B_{y}, \\
& \Sigma F_{z}=0=R F_{z}-H_{z}-B W-B_{z}, \\
& R F_{z}=H_{z}+B W+B_{z},
\end{aligned}
$$

where $R F_{y}$ is the $Y$ reactive force of the right foot; $R F_{z}$ is the $Z$ reactive force of the right foot; $H$, is the total $Y$ hand force; $\mathrm{H}_{2}$ is the total $Z$ hand force; $B W$ is the total body weight; $B$, is the $Y$ body inertial force; and $B_{z}$ is the $Z$ body inertial force.

Moments at the foot during single support were not used by the model and were not needed because there were only two unknowns with two equations.

Double support. Assume:

(1) quasi-static equilibrium;

(2) two arms act as one;

(3) moment arms from heel marker for pushing, from toe marker of rear foot for pulling,

$$
\begin{aligned}
& \Sigma F_{y}=0=L F_{y}+R F_{y}-H_{y}-B_{y}, \\
& \Sigma F_{z}=0=L F_{z}+R F_{z}-H_{z}-B_{z}-B W .
\end{aligned}
$$

Left foot back.

$$
\begin{aligned}
\Sigma M_{x}=0= & -R F_{z} *\left(D F_{y}\right)+\left(B_{z}+B W\right) * D C G_{y} \\
& -B_{y} *\left(D C G_{z}\right)-H_{y} *\left(D H_{z}\right) \\
& +H_{z} *\left(D H_{y}\right)+B_{\mathrm{r}} \\
= & D C G_{y} *\left(B_{z}+B W\right)+D H_{y} *\left(H_{z}\right)+B_{\mathrm{r}} \\
& -D F_{y} *\left(R F_{z}\right)-D C G_{z} *\left(B_{y}\right) \\
& -D H_{z} *\left(H_{y}\right), \\
R F_{z}= & \left(D C G_{y} *\left(B_{z}+B W\right)+D H_{y} *\left(H_{z}\right)+B_{r}\right. \\
& \left.-D C G_{z} *\left(B_{y}\right)-D H_{z} *\left(H_{y}\right)\right) / D F_{z} \\
L F_{z}= & H_{z}+B_{z}+B W-R F_{z}
\end{aligned}
$$

where $L F_{y}$ is the $Y$ reactive force of the left foot; $L F_{z}$ is the $Z$ reactive force of the left foot; $D C G$, is the $Y$ distance from the rear heel to the whole body center of gravity; $D F$, is the $Y$ distance from the rear heel to the $Z$ foot force of other foot; $D H$, is the $Y$ distance from the rear heel to the handle; $D C G_{2}$ is the $Z$ distance from the floor to the whole body center of gravity; $D_{2}$ is the $Z$ distance from the floor to the handle; and $B_{z}$ is the rotational body inertial force.

Similar equations result when the right foot is back for the other portion of double support during right foot stance, with appropriate exchange of right and left foot reactive forces. At this stage, the system is indeterminate because we have three equations $\left(M_{x}, F_{y}\right.$, and $\left.F_{z}\right)$ and four unknowns $\left(L F_{z}, R F_{z}, L F_{m}\right.$ and $\left.R F_{y}\right)$. The solution requires another equation to become determinate; the model assumed that the friction utilization under both feet was the same, and hence the ratios of horizontal $(y)$ to vertical (z) foot forces at each foot were equal (Lee, 1982).

\section{Calculation of trunk muscle forces}

The calculation of L5/S1 compressive forces and the muscle forces contributing to these forces begins with the calculation of abdominal pressure, because this pressure counteracts some of the contraction force of the erector spinae muscles (see Fig. 2). An empirical prediction of abdominal pressure was performed using previously reported equations (Lee et al., 1989; Chaffin and Andersson, 1984) derived from work done by Morris et al. (1961).

The moment arm at which $F_{\text {ABD }}$ acts has been assumed by Chaffin (1975) to vary as the sine of hip angle, with an erect position having a moment arm of $7 \mathrm{~cm}$, increasing to about $15 \mathrm{~cm}$ when stooped over at $\phi_{H}=90^{\circ}$ from vertical (where $\phi_{B}=$ the angle from the hip-to-shoulder link to vertical). The argument that $F_{A B D}$ acts parallel to the compressive force on $\mathrm{LS} / \mathrm{S1}$ was presented by Chafin and Andersson (1984). The line of action of rectus abdominus has also been parallel to the compressive force on $\mathrm{LS} / \mathrm{S} 1$ in other studies (Schultz and Andersson, 1981; Chaffin and Andersson, 1984). This model assumes that all muscle forces act normal to the shear force to create compression only. Reactive shearing forces are then produced by lumbar facet joints, as described in Chaffin and Andersson (1984).

The following equations were used by the model to calculate back and muscle forces (see Fig. 2):

$$
\begin{aligned}
F_{C}= & E S M F+R A M F-F_{A B D} \\
& +\sin (\alpha) *\left(B W_{u}+H_{z}+U B_{z}\right) \\
& +\cos (\alpha) *\left(U B_{y}+H_{y}\right) \\
F_{S}= & \cos (\alpha) *\left(B W_{w}+H_{z}+U B_{z}\right)+\sin (\alpha) *\left(U B_{y}+H_{y}\right)
\end{aligned}
$$

where $F_{c}$ is the L5/S1 compressive force; $F_{s}$ is the L5/S1 shear force due to external forces only (assuming that all muscles act to create compression only); ESMF is the erector spinae muscle force (when resultant moment at LS/S1 was negative), calculated as the resultant moment at LS/S1 divided by the moment arm [0.06 $\mathrm{m}$ for males and females, based on Kumar (1988)]; RAMF is the rectus abdominus muscle force (when resultant LS/S1 moment was positive), calculated as the resultant moment divided by the moment arm [0.10 $\mathrm{m}$ for males and females, from Kumar (1988)]; $F_{A D D}$ is the abdominal force due to intraabdominal pressure; $\alpha$ is the angle from horizontal to LS/S1-shoulder link; $B W_{w}$ is the body weight above 


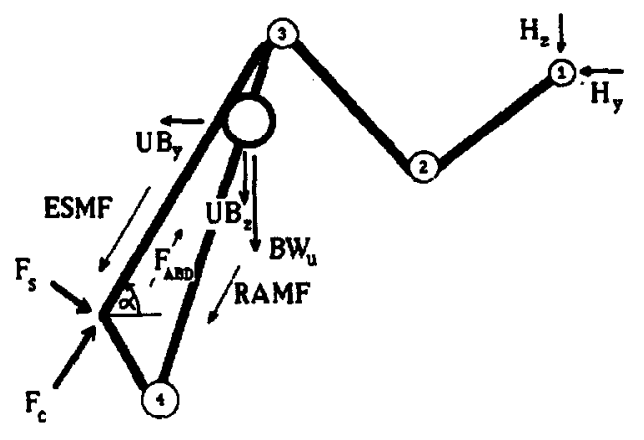

Fig. 2. Free body diagram of the body above the hips, used to calculate LS/S1 shear and compressive forces and erector spinae and rectus abdominus muscle forces. All trunk forces are acting parallel or normal to the compressive force. (Note: it is assumed these muscles act to provide only compression forces on the spinal discs.)

$\mathrm{L} 5 / \mathrm{S} 1 ; U B_{z}$ is the upper body $Z$ inertial force; and $U B_{y}$ is the upper body $Y$ inertial force.

\section{Static calibration experiments}

Linear regressions were formed between the predicted torso muscle forces (erector spinae muscle forces [ESMFs] and rectus abdominus muscle forces [RAMFs]) and the measured RMS-EMGs from both the right and the left groups of these muscles during a gradual increase of push or pull forces. The subject either pushed or pulled in a free static posture. Starting with no exerted hand force, the subject built up to a near maximum push or pull force in a $5 \mathrm{~s}$ period. The regression coefficients were used subsequently to transform dynamic RMS-EMG values to muscle force values to provide a basis for comparison to predicted dynamic muscle force values.

\section{Experimental design and statistical analysis}

A fractional factorial design was used to examine the accuracy and precision of the model predictions as a function of the independent variables, described below. In particular, 20 subjects were selected according to a design which emphasized anthropometric extremes $(<20$ th percentile, $>80$ th percentile for both stature and body weight) and included subjects of 50th percentile stature and weight, with two males and two females in each of the five cells (see Table 1). Healthy young subjects (18-31 yr) volunteered for this experiment. After signing an informed consent form, consistent with University policy, they were weighed and their heights were measured.

Model validation involved three types of analyses: (i) correlations of measured vs predicted variables; (ii) ANOVA analysis of measured and predicted variables; and (iii) ANOVA analysis of residuals formed by taking the difference between measured and predicted values. Schefie multiple comparison tests were performed post hoc, when appropriate.

Linear statistical models were formed for the ANOVA analysis with the following factors (and corresponding levels): subject $(n=20)$, sex (male, female), height (short, average, tall), weight (light, average, heavy), cart resistance (low, high), walking speed $\left(60,100\right.$ steps $\left.\min ^{-1}\right)$, and direction of resistance (push, pull). These factors will be abbreviated SN, SX, HT, WT, CR, WS, and DR, respectively. Only the main effects are presented in this report; first order interactions were not significant.

Table 1. Subject stature and weight descriptions for experimental design to emphasize anthropometric extremes

\begin{tabular}{|c|c|c|c|c|}
\hline & & \multicolumn{3}{|c|}{ Weight (percentile) } \\
\hline & & $<20$ & 50 & $>80$ \\
\hline \multicolumn{5}{|l|}{ Males } \\
\hline \multirow[t]{3}{*}{ Height (percentile) } & $<20$ & $\begin{array}{l}0.170 \mathrm{~m} \\
626.0 \mathrm{~N}\end{array}$ & - & $\begin{array}{c}1.75 \mathrm{~m} \\
885.5 \mathrm{~N}\end{array}$ \\
\hline & 50 & - & $\begin{array}{r}1.78 \mathrm{~m} \\
712.0 \mathrm{~N}\end{array}$ & - \\
\hline & $>80$ & $\begin{array}{r}1.85 \mathrm{~m} \\
689.0 \mathrm{~N}\end{array}$ & E & $\underset{878.0 \mathrm{~N}}{1.84 \mathrm{~m}}$ \\
\hline \multicolumn{5}{|l|}{ Females } \\
\hline \multirow[t]{3}{*}{ Height (percentile) } & $<20$ & $\begin{array}{r}1.54 \mathrm{~m} \\
438.0 \mathrm{~N}\end{array}$ & - & $\begin{array}{r}1.58 \mathrm{~m} \\
845.0 \mathrm{~N}\end{array}$ \\
\hline & 50 & - & $\begin{array}{r}1.62 \mathrm{~m} \\
572.5 \mathrm{~N}\end{array}$ & - \\
\hline & $>80$ & $\begin{array}{c}1.72 \mathrm{~m} \\
569.5 \mathrm{~N}\end{array}$ & - & $\begin{array}{r}1.76 \mathrm{~m} \\
689.0 \mathrm{~N}\end{array}$ \\
\hline
\end{tabular}

Stature and weight percentiles were derived from National Health Survey (1965). There were two subjects in each cell, so only the mean values are given. 


\section{Analytical methods}

The differences between independently measured or derived variables and the same variables predicted by the biodynamic model provided the most critical assessment of model performance. Beyond visual comparisons, one technique to assess differences between predicted and measured quantities was to compare the mean values of the predicted and measured dependent variables for each of the two time-windows (single and total support period for the right leg).

Another method of comparing measured and predicted quantities involved the formation of residuals. The GRF residuals were calculated on a point by point basis throughout the support phase by summing the model predicted force exerted on the foot by the ground and the measured force exerted on the ground by the foot. This was equivalent to subtracting measured values of force exerted on the foot by the ground from the model predicted forces. These residuals were actually intra-subject comparisons. Interpretation of these results requires some explanation: the average represents the sum of the differences between predicted and measured values divided by the number of differences taken. These residual parameters were submitted subsequently to ANOVA analysis to determine which subject and task factors contributed significantly to the errors in the predictions.

\section{Data acquisition hardware}

The cart simulator had handles $0.5 \mathrm{~m}$ apart and oriented so that they were horizontal; hence the subject gripped each handle with the hands prone. The simulator travelled on teflon bushings over aluminum rails, while the cart resistance came from a strap which passed over a variable number of dowels which were affixed to the bottom of the simulator. By changing the tension on this strap, cart resistance varied from 88 to $128 \mathrm{~N}$ (the horizontal force necessary to keep the cart moving at approximately $0.5 \mathrm{~m} \mathrm{~s}^{-1}$ ). The electronics for the portable handles and the EMGs were carried by the cart (see Fig. 3).

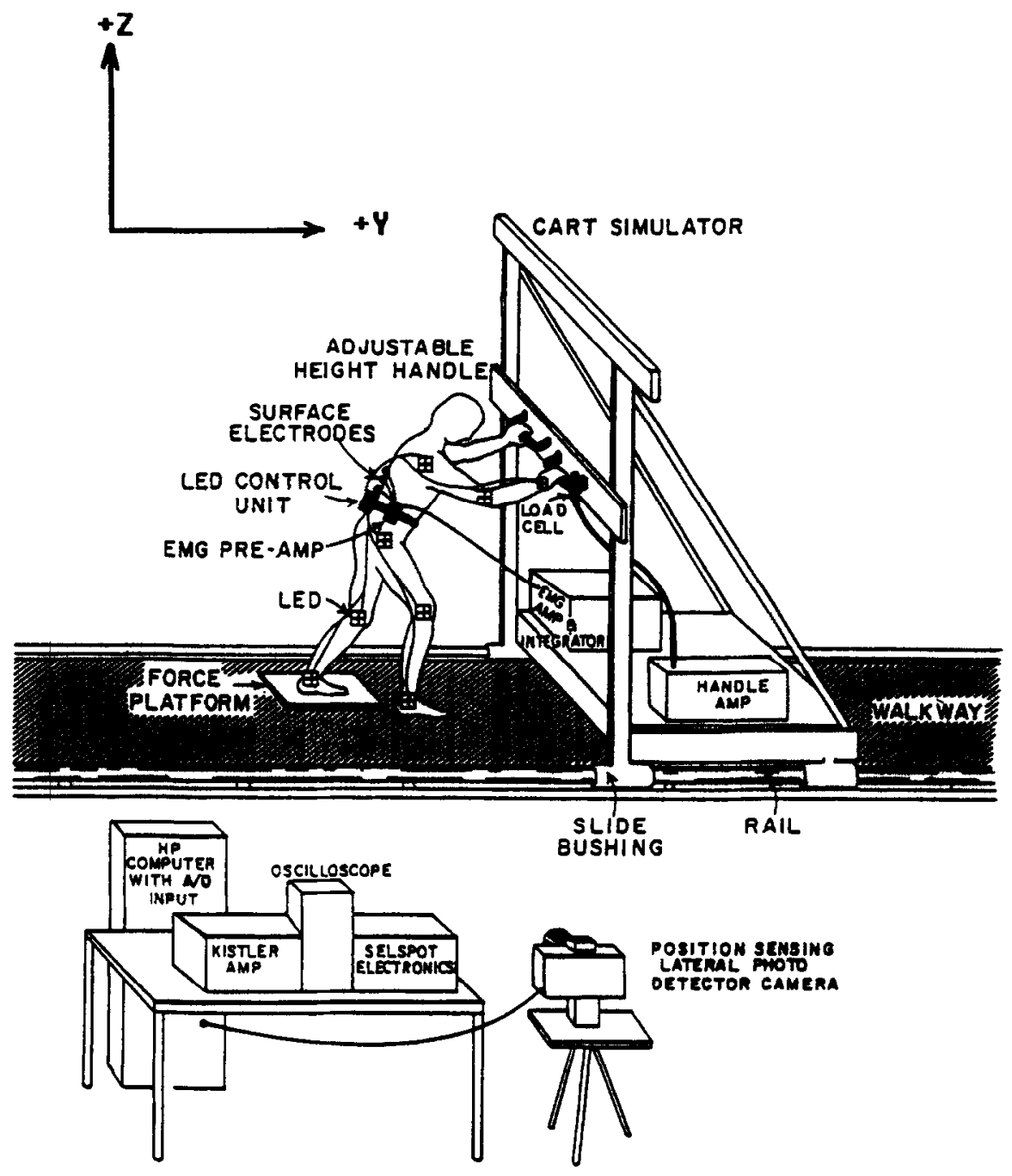

Fig. 3. Diagram of laboratory equipment configuration and the coordinate system used for kinematics and forces exerted on the body. 
Approximated sagittal plane joint center coordinates were sensed by a single camera SELSPOT system (Selcom Selective Electronic Inc., Valdese, North Carolina, U.S.A.) sampling at $50 \mathrm{~Hz}$. The 10 LEDs were placed on the subject as shown in Fig. 1. To prevent distortion the following conditions were placed on LED position detection: (1) the data window was in the central portion of the camera viewing field; (2) lens distortion was compensated for by calibration; and (3) reflection problems were minimized by keeping the reflective laboratory floor out of the viewing window. Hand forces were measured with portable handles which used columns instrumented with strain gauges to detect the horizontal and vertical components of force. The force platform was a Kistler force platform (Kistler Instrumente AG, Winterthur, Switzerland, model 9231A) with six Kistler charge amplifiers (5001) and a Kistler central control unit (5671A). The surface of the force platform was covered with painted plywood, as was the rest of the runway. The static coefficient of friction (COF) of the surface when rubber shoes were worn was approximately 0.7 , while the dynamic COF was about 0.6 (see Andres et al., 1984).

The myoelectrical activity of the torso muscles was detected by bipolar surface electrodes (Hewlett Packard, Andover, Massachusetts, U.S.A., model 14445C disposable electrodes). The electrodes were placed $3 \mathrm{~cm}$ lateral to the midline on both sides of the spine at the $\mathrm{L} 2$ and $\mathrm{L} 3 \mathrm{l}$ level, about $5 \mathrm{~cm}$ apart so that the activity of one side vs the other could be observed. The rectus abdominus activity was recorded by placing the electrodes in a similar manner on the abdomen (centered $3 \mathrm{~cm}$ lateral to the linea alba, above the navel). The electrode signals were sent to preamplifiers in a small box attached to the subject's belt (input impedance $=10^{9} \Omega$, common mode rejection ratio $>120 \mathrm{db}$ ), then to a custom amplifier which converted the raw EMGs to derived RMS values (with a time constant of $55 \mathrm{~ms}$ ) for the four channels of information (left and right erector spinae, and left and right rectus abdominus).

\section{RESULTS}

Figure 4 displays an example of measured vs model predicted results. Quantitative results follow from correlation, ANOVA, and residual analyses.

Correlation analysis of measured us model predicted variables

Both foot force and trunk force variables were compared by forming a simple linear regression between measured and predicted values. Comparisons were made for the average values of the variables over both single and total right leg support phases of the gait cycle (see Table 2). Notice that the vertical foot force during single support had the slope parameter closest to unity. However, for total right leg support the predicted a verage vertical foot force did not correlate nearly as well with its measured counterpart. This implies that predictions during double support (that portion of the total right leg support period excluding single support) were not as valid as those during single support.

Another analysis of the relationships depicts the grand mean of the average value of the variable during single support by subject body weight category for each direction of exertion. The greatest discrepancy emerged for the horizontal GRFs during pushing, with consistent model overprediction. Model overprediction was also apparent for the vertical GRFs during pushing.

Another parameter selected for analysis was the maximum value of the variable within the timewindow, because the direct comparison between measured and predicted values possible with the GRFs may have been more sensitive to changes in maximum values than to average values (see Table 2). Notice again that the slope of the relationship was closest to unity $(0.7)$ for the vertical GRF during single support. Less of the variability in the data was explained by these regressions (using maximum values) compared with the average values shown earlier in Table 2.

Comparisons of the trunk muscle forces derived from RMS-EMGs with model predicted trunk muscle forces indicated that even less of the variability in the data was explained by the simple linear regression model. Most of these regressions were not significant at the 0.05 level. The following section will present

Table 2. Average and maximum GRF correlation results with linear regression coefficients

\begin{tabular}{|c|c|c|c|}
\hline Variable & $R$-square & Intercept & Slope \\
\hline & \multicolumn{3}{|c|}{ Average GRFs } \\
\hline $\begin{array}{l}\text { Single support } \\
R F_{y} \\
R F_{x} \\
C O F\end{array}$ & $\begin{array}{l}0.63 \\
0.66 \\
0.1\end{array}$ & $\begin{array}{c}-10.9 \\
27 \\
0.15\end{array}$ & $\begin{array}{l}0.59 \\
0.93 \\
0.1\end{array}$ \\
\hline \multicolumn{4}{|l|}{ Total support } \\
\hline \multirow[t]{2}{*}{$\begin{array}{l}R F_{y} \\
R F_{z} \\
C O F\end{array}$} & $\begin{array}{l}0.67 \\
0.12 \\
\text { NS }\end{array}$ & $\begin{array}{c}-12.9 \\
357 \\
-\end{array}$ & $\begin{array}{l}0.55 \\
0.26 \\
-\end{array}$ \\
\hline & \multicolumn{3}{|c|}{ Maximum GRFs } \\
\hline $\begin{array}{l}\text { Single support } \\
R F_{\text {, }} \\
R F_{z} \\
C O F\end{array}$ & $\begin{array}{l}0.45 \\
0.45 \\
0.06\end{array}$ & $\begin{array}{r}14.3 \\
164.4 \\
0.27\end{array}$ & $\begin{array}{l}0.3 \\
0.78 \\
0.05\end{array}$ \\
\hline Total support & & & \\
\hline $\begin{array}{l}R F_{y} \\
R F_{x} \\
C O F\end{array}$ & $\begin{array}{l}0.32 \\
0.21 \\
\text { NS }\end{array}$ & $\begin{array}{l}55.4 \\
435 \\
-\end{array}$ & $\begin{array}{c}0.18 \\
0.42 \\
-\end{array}$ \\
\hline
\end{tabular}

$R F_{y}=$ horizontal $G R F, R F_{z}=$ vertical $G R F, C O F=$ ratio of $R F_{y}$ to $R F_{z}$. Notice that the correlations during single support exceeded those from the total support phase, demonstrating the improved performance of the model during single support. Also note that the slope parameters were closest to unity during single support with the vertical GRFs. 
Vertical GRF, pulling

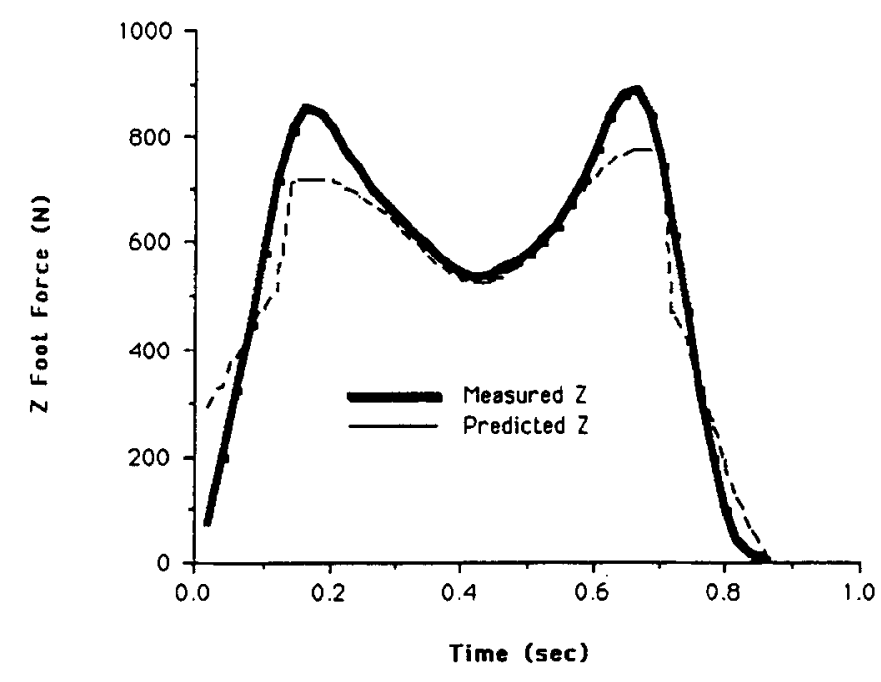

Horizontal GRF, pulling

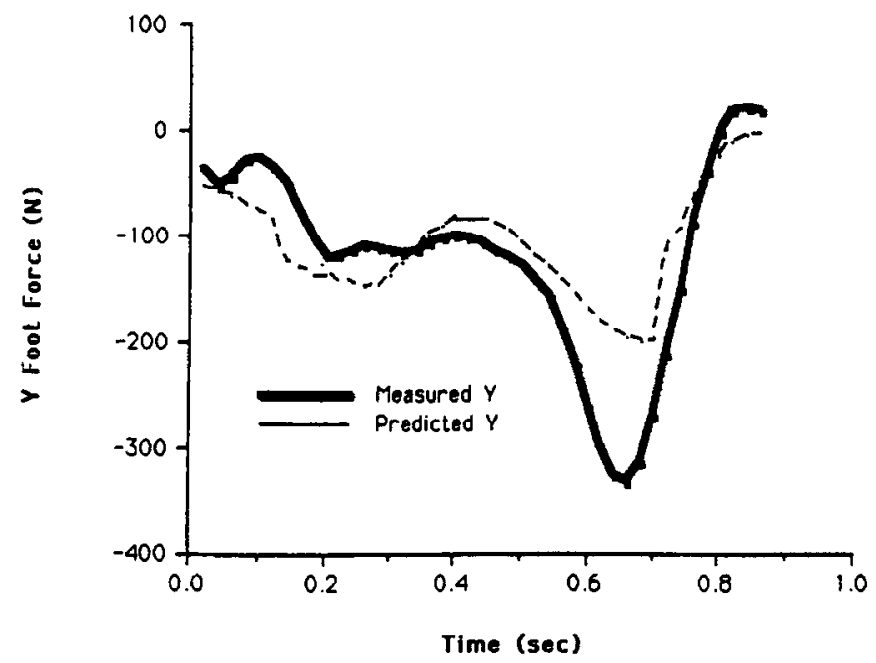

Fig. 4. Measured and predicted ground reaction forces for an example pulling trial. Notice the underprediction of the vertical GRF and the peak horizontal GRF for this particular trial with this subject. This discrepancy was more marked during double support (at either end of the right leg support GRF curves).

more detailed comparisons of derived and predicted trunk muscle forces by performing ANOVA analysis with subject and task factors included in the statistical models.

\section{ANOVA analysis of model predicted variables}

The biodynamic model predicted variables and the measured variables were analyzed with the inclusion of subject (SN, SX, HT, WT) and task (CR, WS, DR) factors. Predicted values exceeded measured values for both horizontal and vertical GRFs, whether for single or total right les support (as seen by comparing grand means in Table 3). Horizontal GRFs were dependent always on direction of exertion, while all vertical GRFs were affected by individual subject factors. Only the mean measured and predicted vertical GRFs during the total right leg support phase had the same factors in the ANOVA analysis.

Predicted trunk muscle forces were subjected to the same analysis (see Table 3). The model underpredicted the ESMFs, but the predicted RAMFs fell between the derived values for the right and left side. Since a wide range of subject anthropometries were purposefully used in these experiments, similar analyses were performed separately for each of the three weight categories (see Table 4). One noticeable difference be- 
Table 3. Average foot and trunk muscle force ANOVA summary

\begin{tabular}{|c|c|c|c|c|}
\hline Variable & $R$-square & Mean & Subject factors & Task factors \\
\hline & \multicolumn{4}{|c|}{ Foot forces } \\
\hline $\begin{array}{l}\text { Single suppor } \\
\text { Meas. } R F_{y} \\
\text { Pred. } R F_{y} \\
\text { Meas. } R F_{z} \\
\text { Pred. } R F_{z} \\
\text { Meas. } C O^{\prime} F \\
\text { Pred. } C O F\end{array}$ & $\begin{array}{l}0.84 \\
0.69 \\
0.66 \\
0.86 \\
0.14 \\
0.14\end{array}$ & $\begin{array}{r}-12.18 \\
1.49 \\
582.15 \\
609.38 \\
1.15 \\
0.29\end{array}$ & $\begin{array}{l}\text { SN } \quad- \\
\text { SN, SX, HT } \\
\text { SN } \\
\quad- \\
-\end{array}$ & $\begin{array}{l}\text { DR } \\
\text { DR } \\
\text { DR } \\
\quad- \\
\quad- \\
\quad-\end{array}$ \\
\hline \multirow[t]{2}{*}{$\begin{array}{l}\text { Meas. } R F, \\
\text { Pred. } R F_{y} \\
\text { Meas. } R F_{*} \\
\text { Pred. } R F_{z} \\
\text { Meas. } C O F \\
\text { Pred. } C O F\end{array}$} & $\begin{array}{l}0.88 \\
0.69 \\
0.8 \\
0.7 \\
0.18 \\
0.29\end{array}$ & $\begin{array}{r}-12.82 \\
1.93 \\
490.71 \\
518.93 \\
0.76 \\
0.22\end{array}$ & $\begin{array}{l}\text { HT } \\
\text { SN } \\
\text { SN, SX, HT } \\
\text { SN, SX, HT } \\
\quad- \\
=\end{array}$ & $\begin{array}{l}\text { WS, DR } \\
\text { DR } \\
- \\
- \\
\text { CR, ws }\end{array}$ \\
\hline & \multicolumn{4}{|c|}{ Trunk forces } \\
\hline Single suppor & & & & \\
\hline $\begin{array}{l}E S M F \\
F M L \\
F M R \\
R A M F \\
F R L \\
F R R\end{array}$ & $\begin{array}{l}0.39 \\
0.73 \\
0.71 \\
0.32 \\
0.36 \\
0.51\end{array}$ & \begin{tabular}{|l|}
561.62 \\
765.2 \\
858.06 \\
71.01 \\
35.48 \\
88.59
\end{tabular} & $\begin{array}{l}\text { SN } \\
\text { SN } \\
\text { SN } \\
\text { SN } \\
\text { SN } \\
\text { SN }\end{array}$ & $\begin{array}{l}\text { CR } \\
\text { DR } \\
\text { DR } \\
\\
= \\
\text { DR }\end{array}$ \\
\hline Total suppor & & & & \\
\hline $\begin{array}{l}E S M F \\
F M L \\
F M R \\
R A M F \\
F R L \\
F R R\end{array}$ & $\begin{array}{l}0.4 \\
0.74 \\
0.69 \\
0.28 \\
0.36 \\
0.51\end{array}$ & \begin{tabular}{r|}
511.23 \\
742.29 \\
860.35 \\
85.79 \\
32.94 \\
88.06
\end{tabular} & $\begin{array}{l}\text { SN } \\
\text { SN } \\
\text { SN } \\
\text { SN } \\
\text { SN } \\
\text { SN }\end{array}$ & $\begin{array}{l}\text { CR } \\
\text { DR } \\
\text { DR } \\
\text { DR } \\
{ }_{\text {DR }}\end{array}$ \\
\hline
\end{tabular}

'Pred.' before the variable denotes the predicted value, and 'Meas.' denotes the measured value, $R F$, $=$ horizontal $G R F, R F_{x}=$ vertical $G R F, C O F=$ ratio of $R F$, to $R F_{z} E S M F=$ erector spinae muscle force predicted by the model, $F M L=$ erector spinae muscle force derived from the RMS-EMG of the left erector spinae muscle, $F M R=$ erector spinae muscle force derived from the RMS-EMG of the right erector spinae muscle, $R A M F=$ rectus abdominus muscle force predicted by the model, $F R L=$ rectus abdominus muscle force derived from left rectus abdominus muscle, and $F R R=$ rectus abdominus muscle force derived from right rectus abdominus muscle. Subject and task factors are explained in the text. Horizontal GRFs all depended on the direction of exertion, with differences in the subject factors involved. Vertical GRFs were affected by more subject factors. The trunk forces all depended on the individual subject factor; the only task factors of importance were the direction of exertion and the cart resistance. Notice that the variability in the measured variables was more readily explained by the selected subject and tasic factors than the variability in the predicted variables.

tween derived and predicted means was that direction of exertion was not a significant factor in the model predicted values. Model predicted mean ESMFs were less than their derived counterparts, most notably for the subjects in the average weight category. The model predicted that mean $R A M F 8$ exceeded the derived values for light and average weight categories, but fell between derived values for the heavy subjects.

\section{Analysis of the differences between measured and model predicted variables}

The residuals between measured and predicted horizontal GRFs were affected by subject, walking speed, and direction of resistance (SN, WS, and DR, as shown in Table 5). The positive residual means for both horizontal and vertical GRFs reaffirm the biomechanical model's bias towards overprediction. The vertical GRF residuals were affected by gender and height (SX and HT). The horizontal to vertical GRF ratio residuals were not explained by the linear statistical model. Average residual values were partitioned by subject body weight category for both single and total right leg support for the horizontal and the vertical GRFs. The horizontal GRF residuals were closest to zero for the subjects in the average weight category $(W T=2)$ when pulling. During pushing the residuals stayed positive across body weight. The vertical GRF residuals were minimized with the light subjects (WT $=1)$ for both pushing and pulling. 
Table 4. Average trunk muscle force ANOVA summary partitioned by subject body weight category

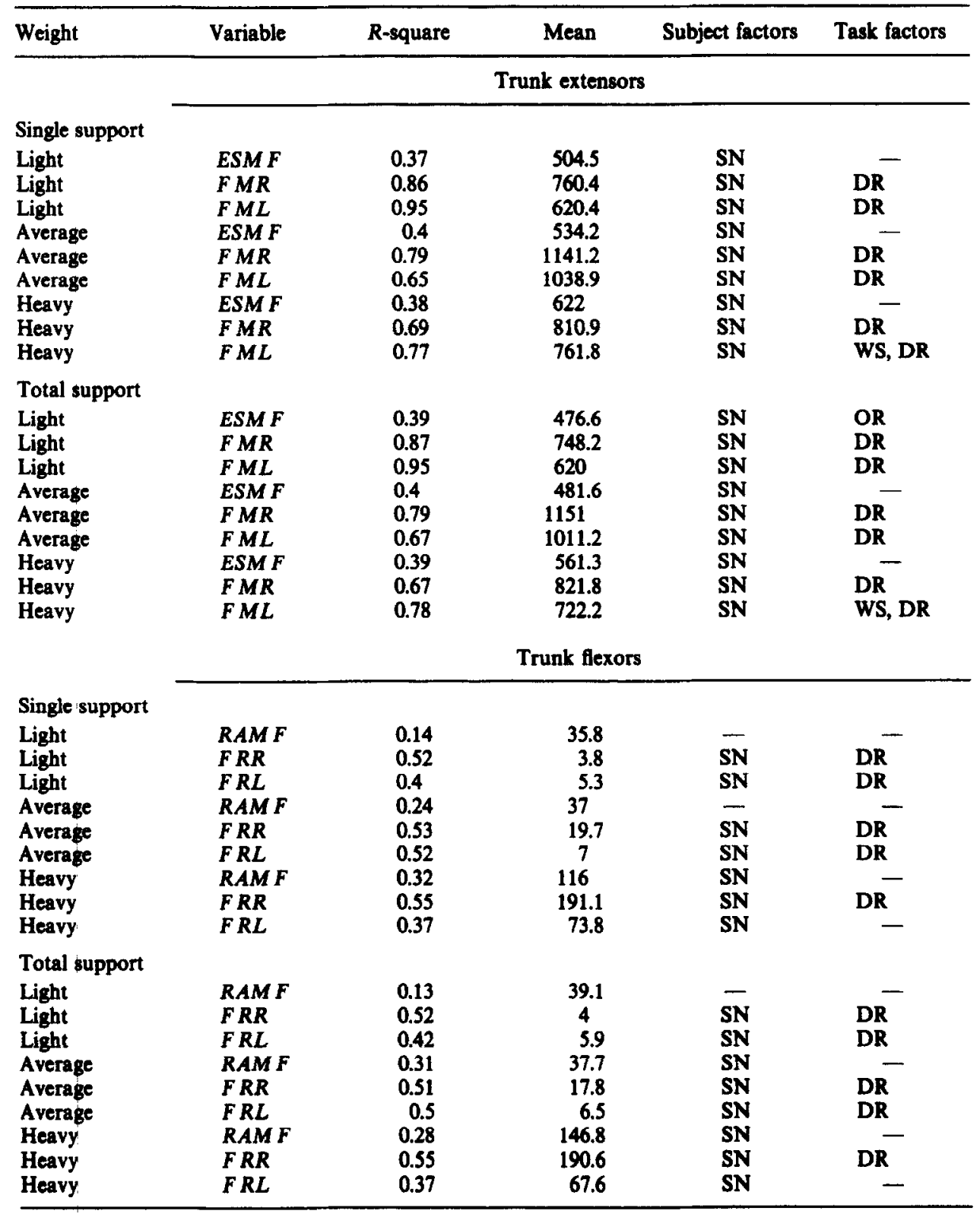

Note that the predicted trunk muscle forces (ESM F and RAM F) were not dependent on the direction of exertion, and that the model underpredicted the ESMF for the subjects of average weight.

Residuals were formed between model predicted trunk muscle forces and derived left and right trunk muscle forces for the total right leg support phase only (Table 5). All of the trunk force residuals were affected significantly by the individual subject factor (SN). Residuals between predicted extensor muscle force and derived muscle forces for each side were large and negative. However, the residuals for the trunk flexors were smaller. Right side ESMF residuals were closest to zero for the heavy subjects. However, on the left side the ESMF residuals were closest to zero for the subjects in the average weight category. Right and left side residual averages for RAMF resembled each other more closely, with minimum residuals for subjects in the average weight category performing pulls.

\section{DIscussion}

The novelty of this particular model is the capability to calculate dynamic parameters that relate to either back overexertion risk or foot slip risk. Previous investigations of pushing and pulling tasks used static analysis, and predictions of internal back forces or ground reaction forces were not attempted. Limitations of the present model will be discussed first in light of the major assumptions required. The effects of 
Table 5. Average GRF and trunk muscle force residual ANOVA summaries

\begin{tabular}{|c|c|c|c|c|}
\hline Variable & $R$-square & Mean & Subject factors & Task factors \\
\hline & \multicolumn{4}{|c|}{ GRF residuals } \\
\hline \multicolumn{5}{|c|}{ Single support } \\
\hline $\begin{array}{l}R F_{y} \\
R F_{z} \\
C O F\end{array}$ & $\begin{array}{l}0.38 \\
0.48 \\
0.09\end{array}$ & $\begin{array}{r}12.74 \\
29.19 \\
-0.34\end{array}$ & $\begin{array}{l}\text { SN } \\
\text { SX, HT } \\
-\end{array}$ & $\begin{array}{l}\text { WS, DR } \\
\text { DR } \\
\quad-\end{array}$ \\
\hline \multicolumn{5}{|c|}{ Total support } \\
\hline \multirow[t]{2}{*}{$\begin{array}{l}R F_{y} \\
R F_{z} \\
C O F\end{array}$} & $\begin{array}{l}0.39 \\
0.6 \\
0.09\end{array}$ & $\begin{array}{r}13.15 \\
31.62 \\
-0.41\end{array}$ & $\begin{array}{l}\text { SN } \\
\text { SN, SX, HT }\end{array}$ & $\begin{array}{c}\text { WS, DR } \\
- \\
-\end{array}$ \\
\hline & \multicolumn{4}{|c|}{ Trunk force residuals } \\
\hline \multicolumn{5}{|c|}{ Total support } \\
\hline $\begin{array}{l}E S M F(\mathrm{~L}) \\
E S M F(\mathrm{R}) \\
R A M F(\mathrm{~L}) \\
R A M F(\mathrm{R})\end{array}$ & $\begin{array}{l}0.29 \\
0.87 \\
0.76 \\
0.75\end{array}$ & $\begin{array}{r}-2785.5 \\
-857.8 \\
-13.1 \\
-20.2\end{array}$ & $\begin{array}{l}\text { SN } \\
\text { SN } \\
\text { SN } \\
\text { SN }\end{array}$ & $\begin{array}{l}- \\
\text { DR } \\
\text { DR } \\
\text { DR }\end{array}$ \\
\hline
\end{tabular}

The positive means for the GRF residuals indicate the model's tendency to overpredict, although these average residuals are small. Note that residuals were larger for the trunk forces.

these assumptions on model performance will then be described. Finally, the validation approach used here will be compared with other validations of biomechanical models.

Model performance can not exceed the quality of the input to the model; hence the input to the model must be considered as a source of discrepancy between measured and predicted values. Sagittal plane joint center position measurement with the SELSPOT realtime position measurement system had two inherent problems: firstly, the location of joint centers was only grossly estimable with markers placed on the skin or clothing at an approximated joint center, as determined by palpation. This problem has been documented extensively before (see Zahedi et al., 1987 for relevant arguments) and will not be probed here. Secondly, the use of a lateral photodetector caused distortion problems due to the lens, the detector, and signal reflection (Gustafsson and Lanshammar, 1977). The steps described in the data acquisition section compensated for these distortions.

Kinematic considerations played a large role in the determination of single vs total right leg support. Model GRF prediction differed depending on the type of support used; without the use of foot switches or a second force plate, the determination of left foot contact with the ground relied on a foot LED floor clearance which had a $1.0 \mathrm{~cm}$ tolerance and a $Y$ coordinate velocity of $<10 \%$ of maximum $Y$ foot velocity. Confounding this determination was the subject's tendency to drag the feet along the floor, perhaps to maximize proprioceptive cues, particularly when going backwards during a pull. Uncertainty in selecting double vs single support could cause dis- continuities in GRF predictions in the transition region.

Several anthropometric variables were based on previous work done on limited samples of cadavers [link lengths and masses from Dempster (1955) and Clauser et al. (1969); radii of gyration from Chandler et al. (1975); diaphragm area from Morris et al. (1961) and Fisher (1967)]. The individual subjects performing the current experiments may not be described accurately by these proportionality constants and average areas, particularly because of the wide range of anthropometries selected. Detailed individual anthropometric measures would have been required to circumvent these innaccuracies, but these were beyond the scope of this study.

The GRF calculations during double support came from moment equilibrium equations. The selection of the reference point for moments taken around the foot-ground interface was an estimate because the center of foot force application in the sagittal plane could not be determined without recording moments from the force platform. Therefore the proper location of the foot contact reference point for horizontal moment arms could have been anywhere between the heel and the toe marker (the model assumed the reference points were the heel marker for pushing and the toe marker for pulling). It was possible that inaccuracies in the horizontal moment arm estimates contributed to model prediction errors during double support.

The other assumption about double support was that the friction utilization was the same under both feet. Since only one force platform was available, this could not be directly verified. When overcoming cart 
inertia (or exerting push or pull forces maximally against a stationary object) most subjects concentrated their foot forces on one foot, using the other foot for balance (Chaffin et al. (1983); also based on observation of the volitional postures and foot forces used by subjects in the static calibration experiments). This concentrated the vertical forces on a smaller surface area, and thus increased the contact pressure at that particular shoe-floor interface. How these static exertions relate to the dynamic case remains unclear.

Since the linear regression coefficients derived during the static calibration experiments were used to transform the RMS-EMGs (obtained during the dynamic experiments) into derived muscle forces for comparison with predicted muscle forces, they played a role in evaluating model performance, and so are discussed here. Muscle activity, as described by EMGs, is highly variable. Processed EMGs (i.e. integrated, average, RMS) have been shown to describe more accuratcly levels of muscle force output than raw EMGs, particularly in isometric situations (see Redfern (1989) for a complete review of these arguments). It is also well known that surface EMGs are susceptible to isignal decrements due to adipose tissue interposed between the sensor and the muscle tissue (Basmajian and DeLuca, 1985). The subjects in these experiments were not of uniform somatotype, and so the quality of surface EMG obtained from the heavy subjects was suspect. Another factor may have been the use of isometric exertions for the calibrations. Most previous work correlating RMS-EMGs with muscle force utilized discrete isometric force levels only (Basmajian and Deluca, 1985), as opposed to our ramp of increasing force.

Vertical and horizontal GRFs were compared for both single and total right leg support because of the sensitivity of model predictions to the transition from one support phase to the other. The most rigorous validation of the model GRF predictions came from the single support phase, since none of the assumptions necessary to make the double support solutions determinate were required.

Measured average horizontal GRF values throughout the total right leg support phase were less than the model predictions. However, inspection of the residual analysis results revealed that larger intra-subject discrepancies existed when the double support phase was included, as opposed to those during single support.

Performing the same comparisons for the vertical GRFs, single support will be discussed first. The amount of variability explained by the regression analyses was similar for measured $(66 \%)$ and predicted (86\%) vertical GRFs. Considering the residuals formed by taking the difference between predicted and measured vertical GRFs during single support, the mean average residual value was $29.2 \mathrm{~N}$, indicating that the model overpredicted more than it underpredicted.

During total right leg support, the mean average values were again greater for predicted than for measured vertical GRFs. The intra-subject comparison disclosed that the mean average force residual for the total right leg support phase was essentially the same as for the single support phase; this implied that the patterns of model over- or underprediction during double support were not consistent from subject to subject, and hence were counteracted when combined across subjects. The statistical consensus was that model predictions of GRFs during double support were not as valid as those during single support. This must be attributed to both the limitations of the moment arms used and the assumption of the equal friction utilization by both feet during double support.

Since the derived ESMFs came from transformations of measured RMS-EMG values with regression coefficients obtained from the static experiments, any limitations in the regressions will be reflected in the derived ESMFs. The ANOVA analysis of derived ESMFs indicated that anywhere from 68 to $74 \%$ of the variability in the data was explained by the independent main factors.

Considering the analysis of residuals formed by subtracting the derived ESMFs from the predicted $E S M F s$, the activity of the right side erector spinae group was predicted better than the activity of the left side muscle group. On an intra-subject basis, the predictions underestimated average ESMFs over the total right leg support phase. The previous discussion of surface EMGs taken from varied somatotypes is also germane, but, given all of these qualifiers, predicted $E S M F$ performed qualitatively the same as right side derived ESMF. It should be stressed that the support phase used throughout this study occurred on the right foot, and therefore it was not surprising that right side derived $E S M F$ s seemed more realistic than left side values; the left side muscles were involved to some degree in the swing phase of the left leg, which occurred during right leg support.

The analysis by subject body weight category provided better insight into the indirect validation of predicted muscle forces compared with muscle forces derived from dynamic RMS-EMGs. Perfect correspondence of derived and predicted values would yield residuals equal to zero. In most cases the ESMF residuals were closest to zero for the medium weight subjects. Adding the consideration that the erector spinae are trunk extensors, they were expected to be most active during pulling. Indeed, in most cases, the residual parameters were closer to zero for the pulling tasks in this investigation.

Derived and predicted $R A M F$ s were examined in a similar manner. Based on the argument that the left side of the muscle group may have some involvement as a synergist during left leg swing, only the right side rectus abdominus muscle group will be discussed here. Minimal anterior muscle activity was seen during the pulling tasks. As was the case with the predicted erector spinae forces, more subject factors (SX, HT, WT) were present in the ANOVA results than for the 
derived forces in the anterior muscles. It should be stressed that the linear regressions derived from the static calibration experiments did not, in general, explain as much of the variability in the RMS-EMGs of the rectus abdominus as for the erector spinae. This was attributed partly to the variation in adipose tissue distribution in the anterior part of the trunk as opposed to the posterior low back region.

The $R A M F$ residual parameters were closest to zero for either the light or the average weight subjects. The rectus abdominus was most active during pushing; i.e. the residual averages during pushing were low. However, residual maxima (all positive) during pushing increased as weight increased, reflecting an increasing model overprediction with heavy subjects.

Lee (1982) also reported much better model performance in predicting GRFs than EMGs. He found that predicted vertical GRFs correlated with measured vertical GRFs $\left(r^{2}=0.65\right)$ and predicted horizontal GRFs correlated with measured horizontal GRFs $\left(r^{2}=0.56\right)$. Mean errors were small $(60$ and $19 \mathrm{~N}$, respectively). This performance was similar to that found in the current investigation. Lee found that there was a subject effect in the differences between predicted and measured GRFs. This was again found in the current investigation in the residual analysis.

The validation approach in this investigation was similar to Lee (1982), with the exception that he used the regression relationships between isometric RMSEMGs and exerted trunk forces to transform predicted dynamic trunk muscle forces to predicted dynamic RMS-EMGs, which were then compared with the measured dynamic RMS-EMGs. Since there have been no other dynamic biomechanical model analyses of pushing and pulling, related validation techniques can only be found in different tasks. Static predictions of mean spine compression acting at $\mathrm{L} 3$ have been correlated with mean intradiscal pressure measurements (Schultz and Andersson, 1981), with a correlation coefficient of 0.91 . This same group later developed a model to predict trunk muscle forces during isometric weight-holding and force resistance tasks (Schultz et al., 1982), which they validated by comparing their calculated trunk muscle tensions with mean myoelectric signal levels. Correlation coefficients ranged from 0.34 to 0.92 , depending on the muscle group and on the function used to predict muscle force. More germane to the current investigation were predictions of dynamic trunk loading; Jager and Luttman (1989) developed a dynamic 19-segment model to assess lumbar stress during load lifting. Their validation consisted of comparing their model calculations with intradiscal pressure measurements taken from the literature; only static holds were compared.

Direct comparisons of predicted and measured ground reaction forces have been performed by others (Pandy and Berme, 1988, 1989). These studies simulated GRFs by assuming joint moment trajectories and performing open-loop (single support) or closed- loop (double support) analyses during normal or pathological walking. However, cart pushing or pulling tasks have not been similarly examined.

Previous biomechanical model validation approaches have not systematically varied subject anthropometry and gender. The evidence from this investigation suggests that this particular dynamic biomechanical model was valid when a wide range of anthropometries was studied. Model predictions, including both GRFs and trunk muscle forces, were better during single support phases than during double support phases.

\section{REFERENCES}

Andres, R. O., Kreutzberg, K. L. and Trier, E. M. (1984) An ergonomic analysis of dynamic coefficient of friction measurement techniques. Technical Report, Center for Ergon: omics, The University of Michigan, Ann Arbor, Michigan.

Ayoub M. and McDaniel, J. (1974) Effects of operators stance on pushing and pulling tasks. AIIE Trans. 6(3), 185-195.

Basmajian, J. V. and DeLuca, C. J. (1985) Muscles Alive, 5th Edn. Williams and Wilkins, Baltimore.

Chaffin, D. B. and Andersson, G. B. J. (1984) Occupational Biomechanics. Wiley, New'York.

Chaffin, D. B., Andres, R. O. and Garg, A. (1983) Volitional postures during maximal push/pull exertions in the sagittal plane. Hum. Factors 25, 541-550.

Chandler, R. F., Clauser, C. E. McConville, J. P., Reynolds, H. M. and Young. J. W. (1975) Investigation of inertial properties of the human body. AMRL-TR-74-137, Aerospace Medical Research Laboratories, Ohio.

Clauser, C. E., McConville, J. T. and Young, J. W. (1969) Weight, volume and center of mass of segments of the human body. AMRL-TR-69-70, Aerospace Medical Research Laboratories, Ohio.

Dempster, W. T. (1955) Space requirements of the seated operator. WADC Technical Report 55-159, University of Michigan.

Dempster, W. T. (1958) Analysis of the two-handed pulls using free body diagrams. J. appl. Physiol. 13, 469-480.

Drillis, R. and Contini, R. (1966) Body segment parameters. Technical Report No. 1166.03, New York University School of Engineering and Science, New York.

Fisher, B. J. (1967) A biomechanical model for the analysis of dynamic activities. M.S.E. thesis, The University of Michigan, Department of Industrial and Operations Engineering, Ann Arbor, Michigan.

Gustafsson, L. and Lanshammar, H. (1977) ENOCH-an integrated system for measurement and analysis of human gait. UPTEC 77-23-R, Uppsala University.

Jackson K. M. (1979) Fitting of mathematical functions to biomechanical data. IEEE Trans. biomed. Engng 28 , 122-124.

Jager, M. and Luttmann, A. (1989) Biomechanical analygis and assessment of lumbar stress during load lifting using a dynamic 9-segment human model. Ergonomics 32, 93-112.

Jager, M., Luttmann, A. and Laurig, W. (1984) The load on the spine during the transport of dustbins. Appl. Ergonomics 15(2), 91-98.

Kroemer, K. H. E. (1969) Push forces exerted in 65 common work positions. Aerospace Medical Research Laboratories Technical Report, USAF AMRL-TR-68-143, Wright Patterson Air Force Base, Ohio.

Kroemer, K. H. E. (1971) Horizontal static forces exerted by men standing in common working positions on surfaces of various tractions. AMRL Report, AMRL-TR-70-114, Aerospace Medical Research Laboratory, Wright Patterson AFB, Ohio. 
Kumar, S. (1988) Moment arms of spinal musculature determined from CT scans. Clin. Biomech. 3(3), 137-144.

Lanshammar, H. (1982) On practical evaluation of differentiation techniques for human gait analysis. J. Biomechanics 14, 99-105.

Lee, K. S. (1982) Biomechanical modelling of cart pushing and pulling. Ph.D. dissertation, The University of Michigan, Ann Arbor, Michigan.

Lee, K. S., Chaffin, D. B., Waikar, A. M. and Chung, M. K. (1989) Lower back muscle forces in pushing and pulling. Ergonomics 32, 1551-1563.

Manning, D. P. (1983) Deaths and injuries caused by slipping, tripping, and falling. Ergonomics 26, 3-10.

Miller, D. I., and Nelson, R. C. (1976) Biomechanics of Sport. Lea and Febiger, Philadelphia.

Morris J. M., Lucas, D. B. and Bresler, B. (1961) Role of the trunk in stability of the spine. J. Bone Jt Surg. 43A, 327-351.

National Health Survey. (1965) Weight, height, and selected body dimensions of adults: 1960-1962. USPHS Publication 1000 , Series 11, No. 8.

Oppenheim, A. V. and Schafer, R. W. (1975) Digital Signal Processing. Prentice-Hall, Englewood Cliffs, New Jersey.

Pandy, M. G. and Berme, N. (1988) Synthesis of human walking: a planar model for single support. J. Biomechanics 21, $1053+1060$.

Pandy, M. G. and Berme, N. (1989) Quantitative assessment of gait determinants during single stance via a threedimensional model-Part 1. Normal gait. J. Biomechanics 22, 717-724.

Plagenhoef, S. C. (1966) Methods for obtaining data to analyze human motion. Res. Q. Am. Ass. Hlth Phys. Ed. 37, 103-112.

Redfern, M. S. (1989) Electromyographic (EMG) signal processing and biomechanical modeling of lower leg muscles. Ph.D. dissertation, Bioengineering Program, The University of Michigan.

Safety Sciences. (1977) Collection and analysis of work surface accident profile data. Final Report for National Institute for Occupational Safety and Health, NTIS access No. PB80-191505.

Schultz, A. B. and Andersson, G. B. J. (1981) Analysis of loads on the lumbar spine. Spine 6, 76-82.

Schultz, A. B., Andersson, G. B. J., Haderspeck, K., Ortengren, R., Nordin, M. and Bjork, R. (1982) Analysis and measurement of lumbar trunk loads in tasks involving bends and twists. J. Biomechanics 15, 669-675.

Snook, S. H., Irvine, C. H. and Bass, S. F. (1970) Maximal weights and workloads acceptable to male industrial workers. Amer. Ind. Hyg. Assoc. J. 31, 79-86.

Strindberg, L. and Peterson, N. (1972) Measurement of force perception in pushing trolleys. Ergonomics 15, 435-438.

Szymusiac, S. M. and Ryan, J. P. (1982) Prevention of slip and fall injuries. Profesional Safety (June) 11-15.

Winkel, J. (1983) On the manual handling of wide-body carts used by cabin attendants in civil aircraft. Appl. Ergonomics 14(3), 186-168.

Zahedi, M. S., Spence, W. D., Solomonidis, S. E. and Paul, J. P. (1987) Repeatability of kinetic and kinematic measurements in gait studies of the lower limb amputee. Prosthet. Orthot. Int. 11, 55-64. 